\title{
Synthesis and Characterization of Star-Shaped Porphyrin-cored Poly(Glutamic Acid) Conjugates as Highly Efficient Photosensitizers
}

\author{
Xiao-Hui Dai ${ }^{*}{ }^{1,2}$, Wei-He Yang ${ }^{2}$, Chuan $\mathrm{Wu}^{2}$, Hua Jin ${ }^{1,3}$, Dan-Dan Chang ${ }^{2}$, Yi-Rong Dai ${ }^{4}$, \\ Jian-Ming Pan $^{2 *}$, and Yong-Sheng Yan ${ }^{1,2}$ \\ ${ }^{1}$ Institute of Green Chemistry and Chemical Technology, Jiangsu University, \\ Zhenjiang 212013, P. R. China \\ ${ }^{2}$ Department of Chemical Engineering, School of Chemistry and Chemical Technology, \\ Jiangsu University, Zhenjiang 212013, P. R. China \\ ${ }^{3}$ School of Chemistry and Chemical Technology, Shanghai Jiaotong University, \\ Shanghai 200000, P. R. China \\ ${ }^{4}$ Hospital Affiliated to Hunan Agricultural University, Zhenjiang 212013, P. R. China
}

\begin{abstract}
Photodynamic therapy (PDT) has drawn wide attention in intensive preclinical and clinical cancer therapy due to its noninvasive nature. A simple and facile synthesis of highly efficient delivery system for photosensitizers (PS) with visualized tumor environment is hence critical. Herein, a simple, safe and promising fluorescent probe, star-shaped poly(glutamic acid) with a porphyrin core (SPPLGA) was synthesized via ring-opening polymerization of $\beta$-benzyl- $L$-glutamate $N$-carboxyanhydride monomer with $5,10,15,20$-tetrakis-(4-aminophenyl)-21H, 23H-porphyrine (TAPP) as the initator, followed by the deprotection of benzyl groups on poly(benzyl- $L$-glutamate). The structure of this novel polymer was thoroughly studied by Nuclear magnetic resonance spectroscopy(NMR), Gel permeation chromatography (GPC) and Fourier transform infrared spectroscopy (FT-IR). Such star-shaped poly(glutamic acid) with porphyrin core could self-assemble into micelles in aqueous solution and exhibit $\mathrm{pH}$-sensitive critical micelle concentration. It was observed that with the decrease in $\mathrm{pH}$, the fluorescence intensity of the SPPLGA increased. On the other hand, the incorporation of poly(glutamic acid) not only improved solubility of porphyrin in water, but also enhanced the production efficiency of the singlet oxygen (about double that of porphyrin). Those results suggest that these polymer, as promising $\mathrm{pH}-$ responsive and tumor-selective photosensitizers, are very promising for PDT and can potentially be used as "theranostics" for future cancer prognosis and therapeutic planning.
\end{abstract}

Keywords: poly (glutamic acid), micelle, self-assembly, PDT, porphyrin

\section{Introduction}

As an emerging noninvasive therapeutic method, photodynamic therapy (PDT) has been considered as a prominent and reliable way of treating cancer in recent years $[1,2]$. PDT employs photochemical interaction between photosensitizers (PS), molecular oxygen and light irradiation of a specific wavelength to generate cytotoxic singlet oxygen $\left({ }^{1} \mathrm{O}_{2}\right)$, in most cases through a type II pathway using $\mathrm{O}_{2}$ as the ${ }^{1} \mathrm{O}_{2}$ source, which cause irreversible damage to malignant cells [3]. Among the numerous photosensitizers, porphyrin and its derivatives are first-line agents in skin, esophagus, lung, head and neck cancer due to their unique property, such as selective accumulation in particular malignant tissues and relatively fast elimination from the body [4]. However, as a result of a photochemical transformation mechanism for PDT, conventional photosensitizers typically (including 
porphyrin and its derivatives) have large -conjugation domains and hydrophobic backbone, and is therefore insufficient to confer excellent solubility in water. Also, the inherent aggregation tendency nature of porphyrin is another important obstacle for practical application in the clinic including toxicity, low bioavailability, leading to induced antitumor therapeutic efficacy [5].

Thus, various delivery systems (e.g. oildispersions, liposomes, polymeric particles, hydrophilic polymer) [6] for porphyrin have been developed to improve solubility of porphyrin while simultaneously mitigating systemic toxicities. Recent work has focused on the possibility of using stimuli-responsive delivery systems [7] to cargo the porphyrin, thereby, (1) reducing the dark toxicity before intravenously administration (2) extending the circulating half-life of porphyrin (3) enabling more administration of porphyrin compound (4) improving the biocompatibility (5) modifying tissue distribution and enhancing intratumoral accumulation (6) controlled sustained drug releases by external or internal stimuli. The last is thought to be triggered by $\mathrm{pH}$, temperature, light, ultrasound, electronic and magnetic fields, biomolecules and enzymes. In general, tumor microenvironment is more acidic $(\mathrm{pH} 6.2-$ 6.9) compared to the normal tissues $(\mathrm{pH} 7.3$ -7.4) [8]. It would thus be desirable to develop a $\mathrm{pH}$ dependent drug delivery system that obtain great superiority in target delivery and sustained release property. Although numerous methods [9-11] have been used to improve the poor solubility of porphyrin, the efficiency of singlet oxygen production remains a challenge.

What's more, porphyrin, an efficient molecular fluorescent probe [12], make it possible to monitor cell uptake and drug delivery pathway in vivo experiments. Combining inherent fluorescent property of porphyrin with selective targeting of a therapeutic agent $\left({ }^{1} \mathrm{O}_{2}\right)$, the so-called "theranostics" [13], provided an important opportunity to investigate the specific procedure of drug release at subcellular level by excellent targeting while facilitating imaging. The gradual acidification of the tumor microenvironment is closely related to innumerable growth factors during the cancer cell metabolism. Therefore, a convenient measurement of $\mathrm{pH}$ value (like fluorescence) is an issue worth of research. Recently, synthetic polypeptide or protein hybrid copolymers [14] have received much attention as most of polypeptide have stimuliresponsive property and abundant group for readily further modification. Also, the former experimental evidence have showed that the conformational structure of polypeptide (helix and -sheet) will control the morphology and size of self-assembled nanoparticles [15]. Furthermore, the study would have been more interesting if the conformational structure also has influence on the photochemical reactivity of porphyrin core. The establishment of real-time in vivo $\mathrm{pH}$ responsive fluorescence polymer would be a promoting work to develop new biocompatible nano-probes to noninvasively visualize the tumor microenvironment.

For those purpose, a pH-responsive nanocarrier has been synthesized for tumor targeting and enhanced photodynamic therapy. As an optimize protocol, the SPPLGA polymer was conveniently prepared by ringopen polymerization (ROP) of $\beta$-benzyl- $L$ glutamate $N$-carboxyanhydride (BLG-NCA), followed by deprotecting of benzyl group. The structure of the as synthesized polymer was characterized by ${ }^{1} \mathrm{H}$ NMR spectroscopy. The micellar properties of the polymer nanoparticles were determined by dynamic light scattering (DLS) and transmission electron microscopy (TEM) analyses. It is found that star-shaped poly $L$-glutamate with a porphyrin core can easily self-assemble into micelles in water and the CMC of polymers can be switched by the $\mathrm{pH}$ value. The relationship between protonation of amino groups of polymer in various $\mathrm{pH}$ value and the intensity of fluorescence of porphyrin core have been evaluated. The singlet oxygen release efficiency of SPPLGA approximately twice as much as porphyrin. The results suggest that these polymers are promising $\mathrm{pH}$-responsive and tumor-selective photosensitizers for PDT and also potentially used as "theranostics" for future cancer prognosis and therapeutic planning.

\section{Experimental}

2.1. Materials and Methods 
Unless stated otherwise, all the reactions were conducted in oven-dried glassware under an atmosphere of nitrogen using anhydrous solvents. THF were purified by distilled from benzophenone and sodium, respectively. $N, N$-dimethylformamide (DMF) was dried over calcium hydride and distilled under reduced pressure, followed by passage through columns of $4 \AA \hat{~ m o l e c u l a r ~ s i e v e s . ~}$ $\mathrm{CH}_{2} \mathrm{Cl}_{2}$ was distilled from calcium hydride. All other commercially obtained reagents were analytical grade or higher and used as received without further purification unless otherwise stated.

5,10,15,20-Tetraphenylporphyrin (TPP) were purchased from Shanghai Dibai Chemical Technology Co., Ltd and used as received. 5,10,15,20-tetrakis-(4-aminopyenyl) $-21 \mathrm{H}, 23 \mathrm{H}$-porphine (TAPP) was synthesized following published procedures [16]. ${ }^{1} \mathrm{H}$ NMR $\left(\mathrm{CDCl}_{3}, \mathrm{ppm}\right): \delta \sim 2.77(\mathrm{~s}, 2 \mathrm{H}$, pyrrole $\mathrm{NH})$, $\delta \sim 5.56\left(\mathrm{~s}, 8 \mathrm{H}, \mathrm{Ph}_{-\mathrm{NH}_{2}}\right), \delta \sim 6.97 \sim 6.99(\mathrm{~d}, 8 \mathrm{H}$, $\operatorname{ArH}), \delta \sim 7.82 \sim 7.84(\mathrm{~d}, 8 \mathrm{H}, \operatorname{ArH}), \delta \sim 8.85(\mathrm{~s}$, $8 \mathrm{H}$, pyrrole- $\beta \mathrm{CH})$.

$\beta$-benzyl- $L$-glutamate $N$-carboxyanhydride (BLG-NCA) was synthesized according to a literature procedure[17]. ${ }^{1} \mathrm{H}$ NMR $\left(\mathrm{CDCl}_{3}\right.$, ppm) : $\delta \sim 2.05 \sim 2.25\left(\mathrm{~m}, 2 \mathrm{H},-\mathrm{CH}_{2} \mathrm{CH}_{2}-\right.$ COO- $), \delta \sim 2.58 \sim 2.63\left(\mathrm{~m}, 2 \mathrm{H},-\mathrm{CH}_{2} \mathrm{CH}_{2} \mathrm{COO}\right.$ $-), \delta \sim 4.34 \sim 4.39(\mathrm{t}, 1 \mathrm{H},-\mathrm{CH}-), \delta \sim 5.13 \sim$ $5.15\left(\mathrm{~s}, 2 \mathrm{H}, \mathrm{PhCH}_{2} \mathrm{O}-\right), \delta \sim 6.17 \sim 6.20(\mathrm{~s}, 1 \mathrm{H}$, $\mathrm{NH}), \delta \sim 7.32 \sim 7.40(\mathrm{~m}, 5 \mathrm{H}, \mathrm{Ph})$.

${ }^{1} \mathrm{H}$ NMR data was obtained by a Bruker (Advance DPX400, Switzerland) spectrometer with $\mathrm{CDCl}_{3}, \mathrm{DMSO}_{6} \mathrm{~d}_{6}$ or $\mathrm{D}_{2} \mathrm{O}$ as solvent at room temperature. The FT-IR spectroscopic measurements were conducted on a Nicolet FT-IR spectrophotometer (Nexus 470, Thermo Electron Corporation) at frequencies ranging from 400 to $4000 \mathrm{~cm}^{-1}$, using $\mathrm{KBr}$ disks at room temperature $\left(25^{\circ} \mathrm{C}\right)$. The molecular weight and molecular weight distribution of polymer were determined on GPC (PerkinElmer Series 200) and a refractive index detector at $30{ }^{\circ} \mathrm{C}$, DMF as the eluent $(1.0 \mathrm{~mL}$ $/ \mathrm{min}$ ), and polystyrene as the calibration standard. TEM micrographs were taken with a JEOL-JEM-2010 (JEOL, Japan) operated at $200 \mathrm{KV}$. A drop of micelle suspension was placed on a copper grid with carbon film for transmission electron microscope (TEM) investigation and stained by a $0.2 \%(\mathrm{w} / \mathrm{v})$ solution of phosphotungstic acid before measurement. The mean size of nanoparticles was determined by dynamic light scattering (DLS) using a Malvern Nano $\mathrm{S}$ instrument (Malvern, UK) consisting of an Autosizer 4700 spectrometer, a pump/filter unit, a model 2013 air-cooler argon ion laser $(75 \mathrm{~mW}$, $488 \mathrm{~nm}$, equipped with a model 2500 remote interface controller, Uniphase), and a computer with DLS software (PCS, version 3.15, Malvern). The measurement temperature was $37{ }^{\circ} \mathrm{C}$, and the measurement angle was $90^{\circ}$. Fluorescence spectrum was performed at room temperature using a Fluorescence spectrophotometer (CaryEclipse, AUS). The spectra were recorded using the excitation mode. Ultraviolet-visible spectroscopy was recorded at room temperature using a Spectrumlab54 UV-visible spectrophotometer.

\subsection{Synthesis of SPPBLG}

Star-shaped Poly ( $\beta$-benzyl- $L$-glutamate) with porphyrin core (SPPBLG) was prepared by ring-opening polymerization (ROP) of BLG-NCA initiated by TAPP in DMF with an appropriate feed ratio. Here is a representative procedure: BLG-NCA $(0.79 \mathrm{~g}$, $3.0 \mathrm{mmol})$ and TAPP $(19.43 \mathrm{mg}, 0.03 \mathrm{mmol})$ were dissolved by anhydrous DMF $(2 \mathrm{~mL})$ in two flame-dried tubes, respectively. Then those two DMF solution were mixed by syringe needle and the polymerization was performed at $50{ }^{\circ} \mathrm{C}$ for 4 days followed by precipitated into $100 \mathrm{~mL}$ anhydrous ether. A dark green solid SPPBLG was obtained after drying under vacuum at room temperature for $24 \mathrm{~h}$. SPPBLG polymers were prepared in a similar manner except that the amounts of BLG-NCA and TAPP were changed according to the targeted molar ratio. The results were shown in Table 1.

\subsection{Synthesis of SPPLGA}

The dark green solid was dissolved in $10 \mathrm{~mL}$ of trifluoroacetic acid and then $3 \mathrm{~mL}$ of $\mathrm{HBr} /$ acetic acid (33 wt $\%$ ) was added. The solution was stirred at $30{ }^{\circ} \mathrm{C}$ for $1 \mathrm{~h}$ before precipitated into excess anhydrous ether and washed twice with anhydrous ether. After drying under vacuum, the precipitate was dialyzed with distilled water and freeze-dried to give the SPPLGA polymer in green powders.

\subsection{Determination of the Critical Micelle Concentrations (CMC)}


The CMC of polymers were determined by employing the hydrophobic dye solubilization method. 1,6-diphenyl-1,3,5-hexatriene (DPH) was used as the hydrophobic probe [18]. A series of aliquots of $0.5 \mathrm{mM}$ DPH methanol solution $(5 \mu \mathrm{L})$ was added to containers and methanol was allowed to evaporate. Then, a concentration gradient of the solution of polymer $(3 \mathrm{~mL})$ were added to containers and capped with teflon film, and then get a series of homogenize solution for about $2 \mathrm{~h}$ at room temperature. UV-vis spectra of samples were recorded in the range of $200-500 \mathrm{~nm}$ range at room temperature.

The experiments were conducted by varying the aqueous polymer concentration in the range of $10^{-5}$ to $1 \mathrm{mg} / \mathrm{mL}$, while keeping the concentration of DPH constant. It is well known that the absorption coefficient of DPH largely depend on its microenvironment. Thus, once DPH transferred from a polar aqueous environment into hydrophobic microenvironment, an abrupt increase in intensity corresponding to the concentration at which micelles are formed can be observed.

\subsection{Micelles Formation}

The self-assembly into micelles of the SPPLGA polymer were carried out by a dialysis method. Briefly, SPPLGA polymer (1 $\mathrm{mg})$ was dissolved in DMF $(1 \mathrm{~mL})$ at an initial concentration of $1 \mathrm{mg} / \mathrm{mL}$. By a microsyringe distilled water was added to the concentration gradually at a speed of 10 $\mu \mathrm{L} / \mathrm{min}$. After a blue tint (water content is about 10-12 wt\%) appears, which indicate the formation of aggregates, another 20-30 wt\% of water was added to stabilize the aggregates. Then micelle will be prepared by dialyzing against distilled water for 3 days, using a dialysis membrane (MWCO 3500). Dynamic light scattering (DLS) and Transmission electron microscope (TEM) were employed to study the morphology of aggregates.

\subsection{Determination of the fluorescence quantum yields}

(TPP) fluorescence quantum yield $\left(\phi_{f}=0.11\right)$ was used as a reference value in dichloromethane solvent $[19,20]$. Fluorescence quantum yields of SPPLGA5 and SPPLGA20 were obtained from the formula (1) and formula (2). The concentration of TPPH2, SPPLGA5 andSPPLGA20 were 1.63 $\mu \mathrm{mol} \cdot \mathrm{L}^{-1}$.

$$
\begin{gathered}
\phi_{f}=0.11 \frac{A_{T P P}}{S_{T P P}} \frac{S_{\text {sample }}}{A_{\text {sample }}} \\
S=\int_{\lambda_{600 n m}}^{\lambda_{800 n m}} I_{f \lambda} d \lambda
\end{gathered}
$$

$A_{T P P}$ : TPP in absorbance at $425 \mathrm{~nm} ; S_{T P P}$ : reference sample TPP integral area of the fluorescence emission spectra from $600 \mathrm{~nm}$ to $800 \mathrm{~nm}\left(\mathrm{E}_{\mathrm{X}}=425\right.$ $\mathrm{nm}) ; A_{\text {sample: }}$ SPPLGA in absorbance at $425 \mathrm{~nm}$ absorbance; $S_{\text {sample }}$ : fluorescence emission spectra of integral area from $600 \mathrm{~nm}$ to $800 \mathrm{~nm}\left(E_{X}=425\right.$ nm). $I_{f \lambda}$ : fluorescence emission curve of $\mathrm{TPPH}_{2}$ or SPPLGA .

\subsection{Singlet oxygen production measurement}

When studying PDT of polymer, the most common method is to detect the ability of singlet oxygen $\left({ }^{1} \mathrm{O}_{2}\right)$ production via a chemical method -monitoring the decrease of the reactive substrate once the polymer generates the ${ }^{1} \mathrm{O}_{2}$. In this study, 1 , 3-diphenylisobenzofuran (DPBF) was chosen as a probe to reveal the singlet oxygen yield, which reacts irreversibly with ${ }^{1} \mathrm{O}_{2}$ that causes a decrease in the intensity of the DPBF absorption band at $456 \mathrm{~nm}$ (TPP as a standard regent) [21,22]. In a typical experiment, the equivalent weight of TPP and SPPLGA $(0.147 \mathrm{mM}, 150 \mu \mathrm{L})$ was mixed with DPBF in DMF $(1 \mathrm{M}, 3 \mathrm{~mL})$ respectively. All the solutions were irradiated with a $650 \mathrm{~nm}$ laser source $(5 \mathrm{~mW})$ and their fluorescence intensity at $456 \mathrm{~nm}$ were recorded with time increase gradually. The intensities of absorption at $456 \mathrm{~nm}$ were plotted against irradiation time. The singlet oxygen quantum yield $(\eta)$ of SPPLGA in DMF was calculated using following equation [23]:

$$
\eta=\phi_{T P P} \frac{t_{T P P}}{t_{S P P L G A}}
$$

$t_{\mathrm{TPP}}$ is the time for decrease in absorption of DPBF in the presence of TPP in DMF solution adjusted to a first-order exponential decay; $t_{\mathrm{SPPLGA}}$ is the time for decrease in absorption of DPBF in the presence of SPPLGA in DMF adjusted to a first-order exponential decay.

\section{Results and Discussion}

3.1. Synthesis of SPPLGA

The synthesis of SPPLGA were by using 


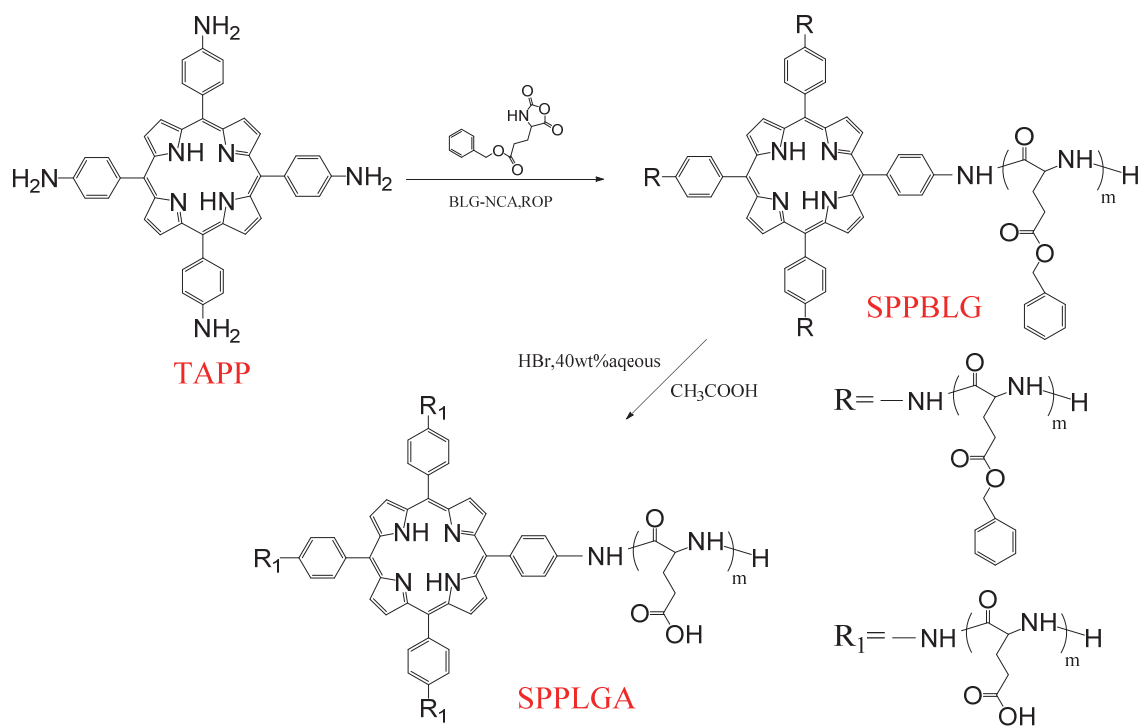

Scheme 1. Synthesis of SPPLGA polymers.

BLG-NCA as monomer for the ring-opening polymerization. The benzyloxycarbonyl group of these compounds was removed by the treatment with hydrobromic acid/acetic acid. The synthetic procedures of SPPLGA polymers were shown in Scheme 1. A series of SPPLGA star-shaped polymers with different segment lengths of the PLGA block were synthesized by varying feed amounts of BLG-NCA. The molecular weights of the polymers are summarized in Table 1.

Table 1. Synthesis of SPPBLG and SPPLGA polymers with porphyrin core.

\begin{tabular}{ccccccc}
\hline Entry $^{\mathrm{a}}$ & {$[\mathrm{M}] /[\mathrm{I}]^{\mathrm{b}}$} & $M_{\mathrm{n}, \mathrm{GPC}}{ }^{\mathrm{c}}$ & $M_{\mathrm{n}, \mathrm{th}^{\mathrm{d}}}$ & $M_{\mathrm{n}, \mathrm{NMR}}{ }^{\mathrm{e}}$ & $M_{\mathrm{w}} / M_{\mathrm{n}}{ }^{\mathrm{c}}$ & $\begin{array}{c}\text { Yield } \\
(\%)\end{array}$ \\
\hline SPPBLG5 & 30 & 9200 & 5100 & 5400 & 1.41 & 64.8 \\
SPPBLG20 & 100 & 34700 & 18200 & 19800 & 1.53 & 56.6 \\
SPPLGA5 & - & 7900 & 3100 & 3300 & 1.40 & 97.4 \\
SPPLGA20 & - & 23600 & 11000 & 13100 & 1.51 & 98.1 \\
\hline
\end{tabular}

a: The numbers after the name of polymer represent the repeating units of polymers at each arm; b: $\mathrm{M}=$ BLG-NCA, I = TAPP; c: $M_{\mathrm{w}} / M_{\mathrm{n}}$ denotes the molecular weight distribution of polymer, where weight-average molecular weight $\left(M_{\mathrm{w}}\right)$ and number-average molecular weight $\left(M_{\mathrm{n}}\right)$ are determined by GPC in THF or DMF. $\mathrm{d}$ : $M_{\mathrm{n}, \mathrm{th}}$ denotes the theoretical number-average molecular weight, $M_{\mathrm{n}, \mathrm{th}}=[\mathrm{M}] /[\mathrm{I}] \times M_{\text {monomer }} \times$ yield $+M_{\text {initiator }}$, molecular weight $\left(M_{\mathrm{w}}\right)$ and number-average molecular weight $\left(M_{\mathrm{n}}\right)$ are determined by GPC in DMF; e: $M_{\mathrm{n}, \mathrm{NMR}}$ was determined from the integral ratio of the signal from the ${ }^{1} \mathrm{H}$ NMR spectra.
The actual polymer molecular weight and chemical structures of SPPLGA star-shaped polymers were characterized by ${ }^{1} \mathrm{H}$ NMR spectroscopy. The ${ }^{1} \mathrm{H}$ NMR spectrums of a representative SPPBLG polymer and SPPLGA polymer are shown in Fig. 1, the characteristic peaks appeared clearly and all signals were marked aside. The methylene groups of glutamate showed their characteristic peaks at 1.6-2.2 ppm, 2.2-2.8 $\mathrm{ppm}$ and 4.2-4.6 ppm. Amide bonds (-NH-) of glutamate showed their characteristic peaks at 8.0-8.3 ppm. The benzene protons of porphyrin showed their characteristic peaks at 7.3-7.6 ppm, 8.0-8.4 ppm, 8.6-9.0 ppm. $M_{n}$, NMR calculated from the ${ }^{1} \mathrm{H}$ NMR spectra was nearly in agreement with $M_{n, \text { th }}$ (Table 1). Those observations confirmed the formation of star-shaped porphyrin-cored SPPBLG. After the deprotection, those strong peak at

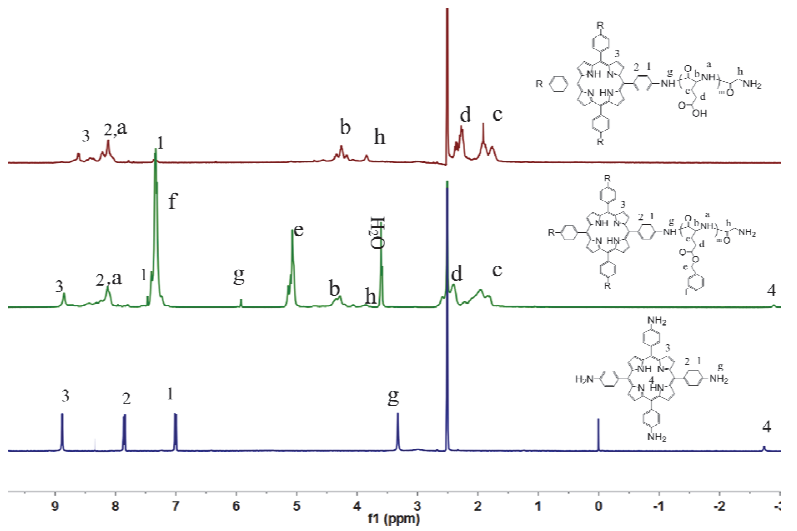

Fig. 1. ${ }^{1} \mathrm{H}$ NMR spectra (DMSO) of a SPPBLG (middle) and SPPLGA (upper) samples. 
7.2-7.5 ppm, 5.0-5.3 ppm, which attributed to the benzyloxycarbonyl group, disappeared in the spectrum of SPPLGA. The absence of aromatic proton peaks and benzyl methylene proton proved the fully debenzylation of the SPPBLG. In all, the above results indicate that star-shaped SPPLGA polymers were successfully synthesized from the ring-opening polymerization of BLG-NCA using a tetra-aminophenyl terminated porphyrin initiator at $50{ }^{\circ} \mathrm{C}$, followed by deprotection of benzyloxycarbonyl group. Figure 2 shows the typical GPC curves (DMF solvent) of as-synthesized SPPBLG and SPPLGA polymers. Notably, unimodal trace has been shown in GPC, suggesting that narrow molecular weight distribution polymers were formed. The GPC results of unimodal peak at different elution times denote a progression of the polymer molecular weight.

Also the structure of the prepared polymers was confirmed by FT-IR. As exhibited in Fig. 3 , a strong absorbance at $1730 \mathrm{~cm}^{-1}$ contributed to the stretch vibration of $\mathrm{C}=\mathrm{O}$ in SPPBLG or SPPLGA was observed in all the polymers. In addition, the absorbance of carbonyl amide groups appeared at $1630 \mathrm{~cm}^{-1}$, while the stretching vibration of the amino $\mathrm{N}-\mathrm{H}$ appeared at $3290 \mathrm{~cm}^{-1}$. Compared with SPPBLG, disappearance of absorbance at 690 and $750 \mathrm{~cm}^{-1}$ in the IR spectra of SPPLGA polymers, which belongs to vibration of benzene of benzyloxycarbonyl group, indicated that SPPLGA polymers without benzyloxycarbonyl group was successfully synthesized.

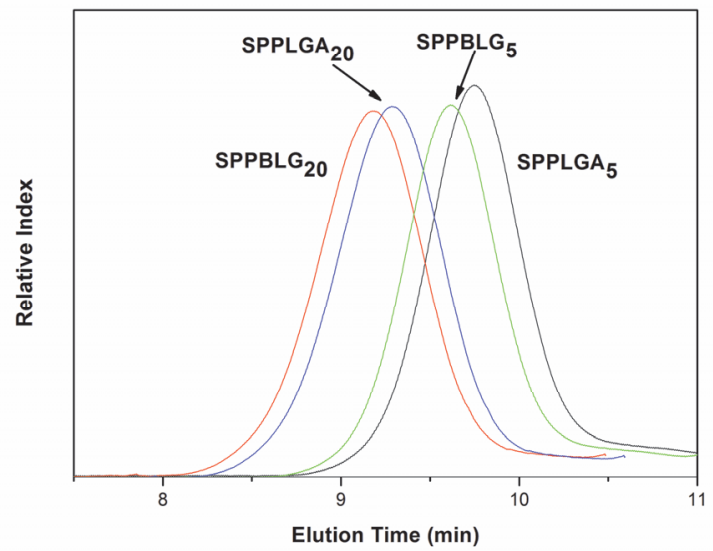

Fig. 2. GPC traces of the SPPBLG and SPPLGA samples.

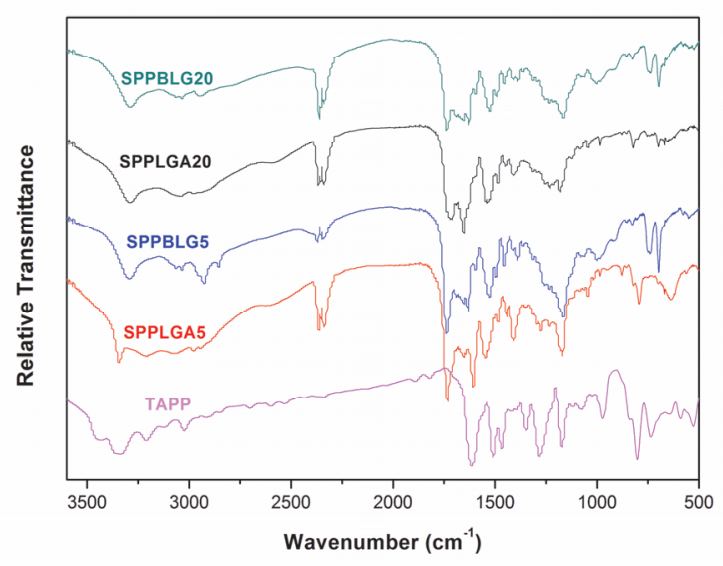

Fig. 3. FT-IR spectra of SPPBLG and SPPLGA samples.

\subsection{Self-Assembly Behavior of SPPLGA}

To study the self-assembly behavior of the SPPLGA in aqueous solution, dye solubilization method was used to verify the CMC determination with a serial of different concentrations DPH [24]. Once DPH was preferentially partitioned into the hydrophobic core of the micelles, an abrupt increase in absorbance intensity can be observed with increasing polymer concentrations. The CMC was determined by extrapolating the absorbance versus logarithmic concentration.

As shown in Fig. 4A, the intensity of the absorption spectra of DPH versus the logarithm of the SPPLGA polymer concentrations was plotted. It can be seen that the intensity was almost constant at relatively low concentration. With the concentration of the polymer increase, an abrupt increase in absorbance intensity was observed, indicating the incorporation of DPH in hydrophobic micellar core as well as the formation of micelles. The CMC values determined by dye solubilization method for the water-soluble polymers are $44.9 \mu \mathrm{g} / \mathrm{mL}$ (SPPLGA5) and $50.1 \mu \mathrm{g} / \mathrm{mL}$ (SPPLGA20), respectively, indicating that $\mathrm{CMC}$ increased with increasing hydrophilic segment. To have a better understanding on the role of pH-sensitivity of polymer, we also examined the $\mathrm{CMC}$ in aqueous solutions with different $\mathrm{pH}$ value. 

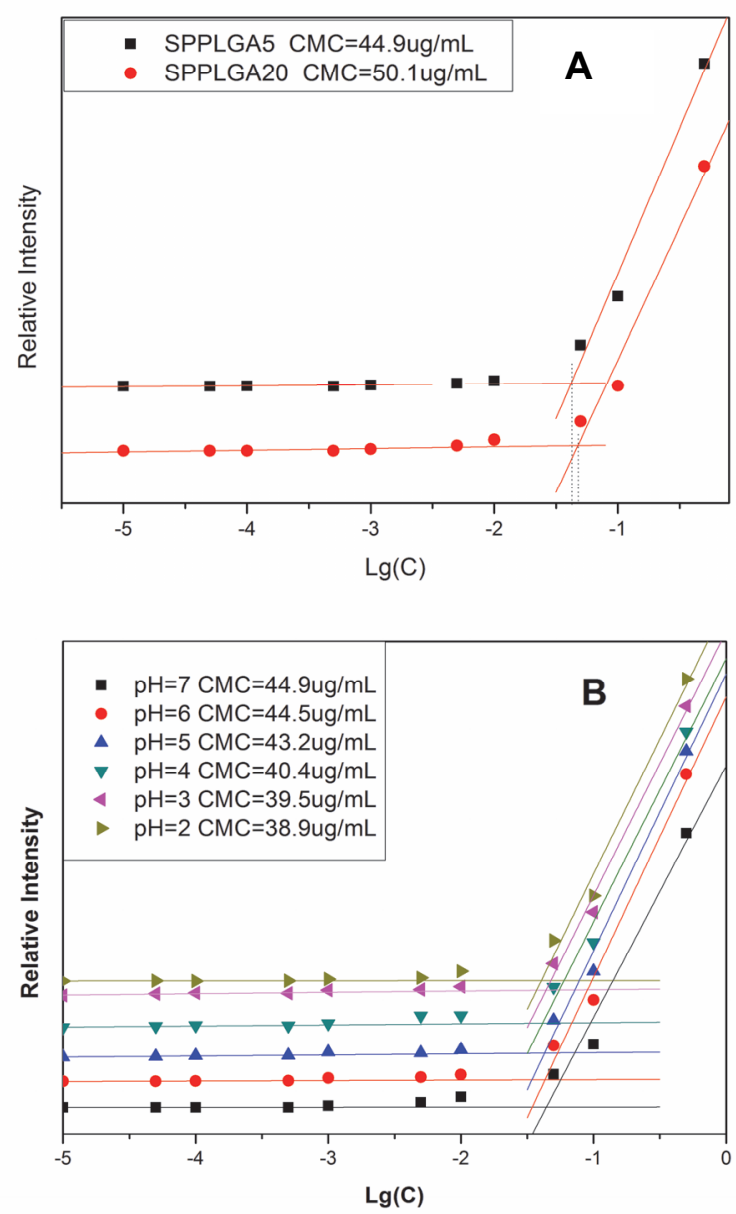

Fig. 4. (A) The relationship of the absorbance intensity of DPH at $334 \mathrm{~nm}$ vs the concentration of SPPLGA samples at $25^{\circ} \mathrm{C}$. (B) Intensity of DPH excitation spectra monitored at $334 \mathrm{~nm}$ as a function of polymer concentrations of SPPLGA5 samples with different $\mathrm{pH}$.

As shown in Fig. 4B, the CMC of SPPLGA5 polymer increased with the increasing $\mathrm{pH}$ value, suggesting that the self-assembly of SPPLGA was greatly dependent on the $\mathrm{pH}$ value. The CMC showed an increase trend from 38.9 to $44.9 \mu \mathrm{g} / \mathrm{mL}$ when the $\mathrm{pH}$ rise from 2 to 7 , owing to the ionization of pendant carboxylic acid group in those polymers. There is a positive correlation between the $\mathrm{pH}$ value and the protonated states of carboxylic acid group. The lower $\mathrm{pH}$ value, the more carboxylic groups of polymers were hydrolyzed, leading to the arms of polymers relatively more hydrophobic. That is to say, those hydrophobic pedant neutral molecular displayed an increasing electrostatic repulsions under the acidic environment. As the CMC of polymers will decrease with the more hydrophobic group of polymers (i.e. more neutral pendant carboxylic acid group). There is no surprise that the lower CMC value were observed under more acid environment. At same time, under the neutral or high $\mathrm{pH}$ polymers with more pendant carboxylic acid group (SPPLGA20) could release more protons to show more hydrophilic properties, leading to a relatively higher CMC value than the short segment polymers (SPPLGA20).

The morphology of self-assembled micelles was confirmed by TEM. As shown in Fig. 5A, it is evident that regular spherical shape and well-dispersed self-assembled micelles were mainly presented for SPPLGA polymers. The sizes of micelles from the TEM image were $\sim 40 \mathrm{~nm}$ and DLS revealed that these geometries maintained a hydrodynamic radius of $107 \mathrm{~nm}$ (Fig. 5B). The reason for difference in size observed by TEM and DLS could be related to the fact that the TEM reveals morphology size in dehydrated solid state, whereas the DLS measures the hydrodynamic diameter in an aqueous environment. Compared to the DLS sample, dehydration and subsequent collapse of the polymer chain for TEM sample could lead to a smaller diameter. Similar difference in size as a result of different measuring techniques was also made in other papers [25-27].
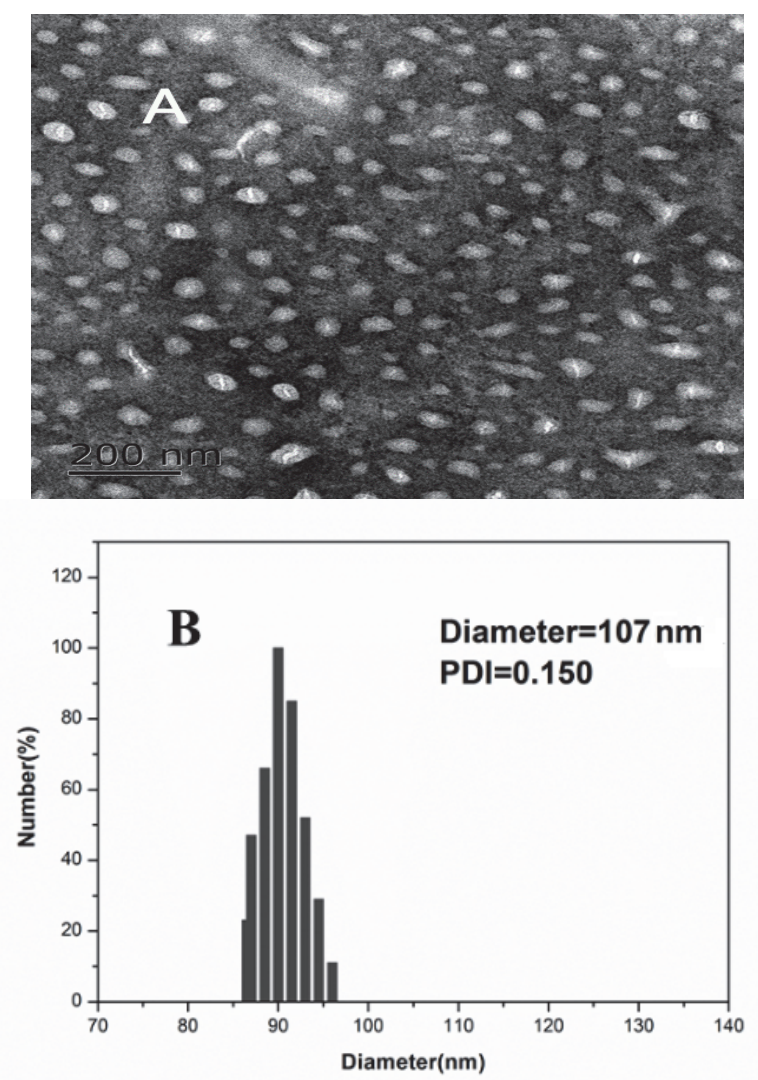

Fig. 5. (A) TEM photographs of SPPLGA20 polymers (B) Size distribution of SPPLGA20 polymers. 
3.3. Electronic Absorption and Photophysical Properties.

The electronic absorption and basic photophysical properties for SPPLGA were characterized in DMF by UV-vis spectroscopy (Fig. 6A). All the SPPLGA gave typical absorption spectra, showing the Soret-band at $425 \mathrm{~nm}$, Q-band at $500-700 \mathrm{~nm}$. Relative to free porphyrin, the red-shifts and increased intensity of Soret-band of polymers indicated that polymer chains induced distortion of porphyrin core. The very similar Q-band absorptions indicated that the luminescent property of porphyrin moiety was not perturbed by the outer polymers chain, which would potentially qualified SPPLGA as an efficient biological probe and photosensitizer for PDT. In addition, with the increase of polymer chain length, the maximum absorption wavelength and the molar absorption coefficient of the SPPLGA increased. Upon excitation with various concentrations of polymers, the very sharp B-band strictly followed the Lambert-Beer law, suggesting that aggregation was not significant for those polymers (Fig. 6B) $[28,29]$.

In addition, the fluorescence quantum yields of $\left(\phi_{f}\right)$ these polymers were investigated based on formula (1) and formula
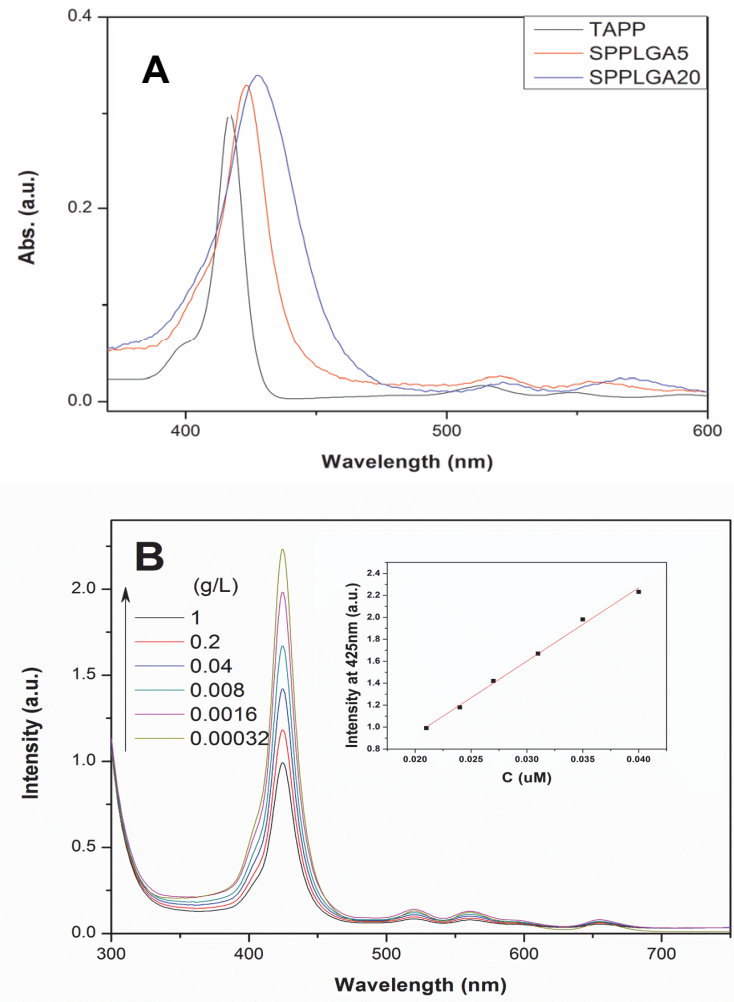

Fig. 6. UV-vis spectra of (A) TAPP and SPPLGA, (B) SPPLGA at different concentrations in DMF. The inset plots the Q- band absorbance at $425 \mathrm{~nm}$ versus the concentration of SPPLGA.
(2). The fluorescence quantum yields of SPPLGA5 and SPPLGA20 was 0.54 and 0.63 , respectively. The high fluorescence quantum yields may be attributed to the resonance interaction between porphyrin core and polypeptides [30]. Also rigid chain of polymer arm prevented self-aggregation and self-quenching of the singlet excited state of the porphyrin core through high steric hindrance effect [31].

It is known that conventional photosensitizers appeal to aggregate in aqueous media as a result of their-interactions and lipophilic nature. But the polymer as synthesized are quite soluble in water because the amino groups are protonated under this condition. Herein, we investigated the fluorescence spectrum in water in the presence of a trace amount of organic solvents $[0.1 \%$ tetrahydrofuran (THF)] under different acid intensity. Obviously, we found out that there is a possible link with the max fluorescence intensity of polymer and $\mathrm{pH}$ value. As shown in Fig. 7, all the polymers_showed similar spectral features, which indicated that they were essentially non-aggregated under an acidic or neutral condition $(\mathrm{pH}<8)$. Contrary to the decrease in $\mathrm{pH}$ of aqueous solution, there was a growing tendency of fluorescence intensity, which may be relative to the fact that carboxy groups of polymer were more protonated at lower $\mathrm{pH}$ value resulted in reduced polymer aggregation. And the aggregation of porphyrin has been proven to be more chance for intramolecular photoinduced electron transfer (PET) progressing [32], which means the fluorescence intensity of porphyrin would be quenched at higher $\mathrm{PH}$ value. As the range of intracellular $\mathrm{pH}$ is about 4.5-7.4 [33], those polymers with remarkable $\mathrm{pH}$-dependent fluorescence represent potential application for intracellular $\mathrm{pH}$ imaging.

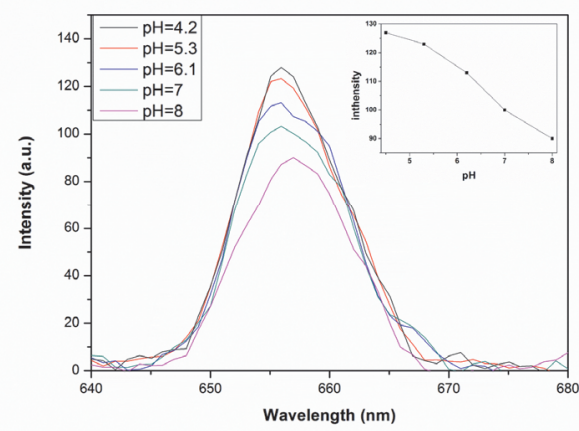

Fig. 7. Fluorescence spectra of SPPLGA20 $(2 \mu \mathrm{M})$ in citrate buffer solutions with different $\mathrm{pH}$ (excited at 610 $\mathrm{nm})$. The inset plots the variation of the relative fluorescence intensity versus the $\mathrm{pH}$ value. 


\subsection{Singlet oxygen production measurement}

To evaluate the ability of these polymer to generate singlet oxygen, the basis of the photoinactivation process, was qualitatively estimated by a steady-state method using 1, 3-diphenylisobenzofuran (DPBF) as the scavenger. DPBF is a singlet oxygen quencher which can qualitatively establish the production of singlet oxygen, which result in a decreased intensity of the DPBF fluorescence band at $414 \mathrm{~nm}$. According to the reported singlet oxygen generation estimation method, The singlet oxygen quantum yield $(\eta)$ of polymer were evaluated by comparison with those obtained in previous work for 5,10,15,20tetraphenylporphyrin (TPP) $(0.67 \pm 0.09)$ [23].

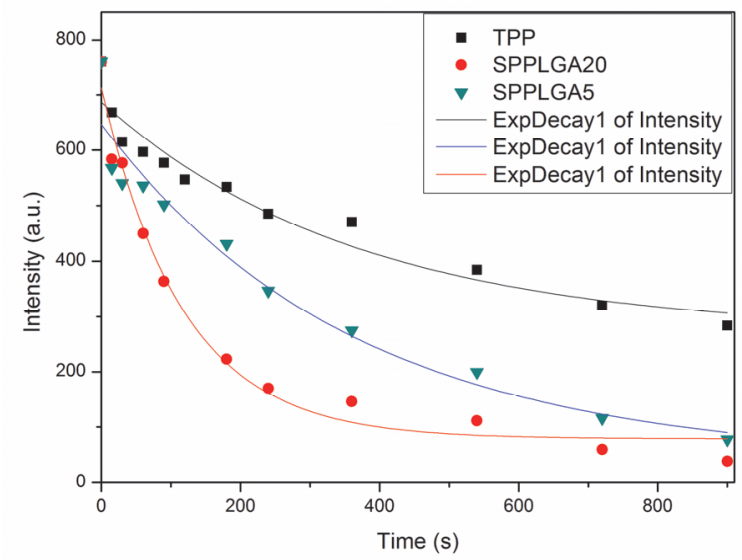

Fig. 8. Fluorescence intensity at $456 \mathrm{~nm}$ decay curves of DPBF as a function of time with a laser source $(650 \pm 10 \mathrm{~nm}, 5 \mathrm{Mw})$ with adding TPP (ם), SPPLGA20 ( ) and SPPLGA5 ( $\boldsymbol{\nabla})$, respectively.

In Fig. 8, the fluorescence intensity of DPBF of all the polymers decreased upon the time and the decay curves of all polymers were quite comparable to TPP. However, the DPBF decay rate of polymer was much higher than TPP, denoting the polymer in this case exhibited a higher efficiency to generate singlet oxygen under the same experimental conditions than TPP. This high efficiency may be attributed to PET between porphyrin and poly (glutamic acid). This results agreed with observations that electron transfer between Alexa fluorophores and natural amino acids [30]. Comparing the DPBF decay rate of polymers with different arm length, we observed that polymers with long arm length SPPLGA20 were also an efficient photosensitizer. The singlet oxygen quantum yield $(\eta)$ of SPPLGA5 and SPPLGA20 in DMF solution as calculated from the eq. 3 (see Experimental Section) were 1.12 and
1.01, respectively. This different values may indicate a dependence on the relative orientations (steric factor) of peptide and porphyrin core [34]. Thus, the effective generation of singlet oxygen suggests that the SPPLGA presented here is a promising candidate as PDT agents. With the fluorescence imaging of porphyrin, these systems not only selectively and efficiently destroy the targeted malignancy tissue on laser treatment, but also could real-time monitor the distribution patterns of drugs.

\section{Conclusion}

We developed a functionalization strategy for porphyrin by incorporation with poly(glutamic acid) (SPPLGA) via ringopening polymerization of $\beta$-benzyl- $L$ glutamate $N$-carboxyanhydride monomer, followed by the deprotection of benzyl groups on poly(benzyl- $L$-glutamate). Such starshaped polypeptide can self-assembly to micelles in an aqueous environment. In the meantime, the in further studies showed there were positive relationships between the CMC and the $\mathrm{pH}$ value. On the other hand, the incorporation of poly (amino acid) not only improved solubility in water of photosensitizer (porphyrin), but also substantially enhanced the singlet oxygen efficiency. It is found that the polymer showed remarkable different fluorescence intensity in the environments for tumors and normal tissue. Those results suggest that these polymers are promising $\mathrm{pH}$-responsive and tumor-selective photosensitizers for PDT and also potentially used as "theranostics" for future cancer prognosis and therapeutic planning.

\section{Acknowledgement}

The authors are greatly grateful for the financial support of the National Natural Science Foundation of China (21004031), the Natural Science Foundation of Jiangsu Province (BK2011459), and the Practice Innovation Program of Jiang Su (SJZZ-0138).

\section{References}

1. M. D. Daniell and J. S. Hill, Aust. NZ J. Surg., 61 (1991) 340.

2. P. Agostinis, K. Berg, K. A. Cengel, T. H. Foster, A. W. Girotti, S. O. Gollnick, S. M. Hahn, M. R. Hamblin, A. Juzeniene and D. 
Kessel, CA Cancer J. Clin., 61 (2011) 250.

3. D. E. J. G. J. Dolmans, D. Fukumura and R. K. Jain, Nat. Rev. Cancer, 3 (2003) 380 .

4. M. Ethirajan, Y. Chen, P. Joshi and R. K. Pandey, Chem. Soc. Rev., 40 (2011) 340.

5. A. E. O'Connor, W. M. Gallagher and A. T. Byrne, Photochem. Photobiol., 85 (2009) 1053.

6. E. Paszko, C. Ehrhardt, M. O. Senge, D. P. Kelleher and J. V. Reynolds, Photodiagnosis Photodyn. Ther., 8 (2011) 14.

7. C. K. Lim, J. Heo, S. Shin, K. Jeong, Y. H. Seo, W. D. Jang, C. R. Park, S. Y. Park, S. Kim and I. C. Kwon, Cancer Lett., 334 (2013) 176.

8. S. S. Han, Z. Y. Li, J. Y. Zhu, K. Han, Z. Y. Zeng, W. Hong, W. X. Li, H. Z. Jia, Y. Liu, R. X. Zhuo and X. Z. Zhang, Small, (2015), DOI: 10.1002/small, 201402865.

9. X. L. Liang, X. D. Li, X. L. Yue and Z. F. Dai, Angew. Chem. Int. Ed., 50 (2011) 11622 .

10. J. Li, W. Zhang, Z. Hu, X.-J. Jiang, T. Ngai, P.-C. Lo, W. Zhang and G. Chen, Polym. Chem., 4 (2013) 782.

11. S. S. Lucky, K. C. Soo and Y. Zhang, Chem. Rev., 115 (2015) 1990.

12. J. Shi, T. W. Liu, J. Chen, D. Green, D. Jaffray, B. C. Wilson, F. Wang and G. Zheng, Theranostics, 1 (2011) 363.

13. Z. Sheng, D. Hu, M. Zheng, P. Zhao, H. Liu, D. Gao, P. Gong, G. Gao, P. Zhang, Y. Ma and L. Cai, ACS Nano, 8 (2014) 12310.

14. C. He, X. Zhuang, Z. Tang, H. Tian and X. Chen, Adv. Healthc. Mater., 1 (2012), 48.

15. Y. Shen, X. Fu, W. Fu and Z. Li, Chem. Soc. Rev., 44 (2015) 612.

16. L. Zhang, Y. Lin, Y. Zhang, R. Chen, Z. Zhu, W. Wu and X. Jiang, Macromol. Biosci., 12 (2012) 83.

17. Y. Chen and C.-M. Dong, J. Phys. Chem. B, 114 (2010) 7461.

18. D. E. Discher and F. Ahmed, Ann. Rev. Biomed. Eng., 8 (2006) 323.

19. T. B. Ren, A. Wang, W. Z. Yuan, L. Li and Y.
Feng, J. Polym. Sci. Part A: Polym Chem., 49 (2011) 2303.

20. Z. Y. Li, H. Y. Wang, C. Li, X. L. Zhang, X. J. Wu, S. Y. Qin, X. Z. Zhang and R. X. Zhuo, J. Polym. Sci. Part A: Polym Chem., 49 (2011) 286.

21. S. M. Peng, Y. Chen, C. Hua, C.-M. Dong, Macromolecules, 42 (2008) 104.

22. M. Wozniak, F. Tanfani, E. Bertoli, G. Zolese and J. Antosiewicz, BBA-Lipid Lipid Met., 1082 (1991) 94.

23. D. B. Tada, L. L. R. Vono, E. L. Duarte, R. Itri, P. K. Kiyohara, M. S. Baptista and L. Rossi, Langmuir, 23 (2007) 8194.

24. I. Astafieva, X. F. Zhong and A. Eisenberg, Macromolecules, 26 (1993) 7339.

25. Y. Y. Li, X. Z. Zhang, H. Cheng, G. C. Kim, S. X. Cheng and R. X. Zhuo, Biomacromolecules, 7 (2006) 2956.

26. C. Giacomelli, V. Schmidt and R. Borsali, Langmuir, 23 (2007) 6947.

27. M. Pinna, S. Hilt1, X. Guo, A. Böker and A. V. Zvelindovsky, ACS Nano, 4 (2010) 2845.

28. R. Ideta, F. Tasaka, W. D. Jang, N. Nishiyama, G. D. Zhang, A. Harada, Y. Yanagi, Y. Tamaki, T. Aida and K. Kataoka, Nano Lett., 5 (2005) 2426.

29. H. Gu, K. Xu, Z. Yang, C. K. Chang and B. Xu, Chem. Commun., 34 (2015) 4270.

30. H. Chen, S. S. Ahsan, M. E. B. SantiagoBerrios, H. D. Abruña and W. W. Webb, J. Am. Chem. Soc., 132 (2010) 7244.

31. H. Deng, Y. Su, M. Hu, X. Jin, L. He, Y. Pang, $\mathrm{R}$. Dong and $\mathrm{X}$. Zhu, Macromolecules, 48 (2015) 5969.

32. X. J. Jiang, P. C. Lo, S. L. Yeung, W. P. Fong and D. K. Ng, Chem. Commun., 46 (2010) 3188.

33. B. Tang, F. Yu, P. Li, L. Tong, X. Duan, T. Xie and X. Wang, J. Am. Chem. Soc., 131 (2009) 3016.

34. D. E. Williams, E. A. Dolgopolova, P. J. Pellechia, A. Palukoshka, T. J. Wilson, R. Tan, J. M. Maier, A. B. Greytak, M. D. Smith, J. A. Krause and N. B. Shustova, J. Am. Chem. Soc., 137 (2015) 2223. 